\title{
Author Correction: Generation and characterization of ultrathin free-flowing liquid sheets
}

Jake D. Koralek1, Jongjin B. Kim (10) 1, Petr Brůža (1) 2,3, Chandra B. Curry (1) 1,4, Zhijiang Chen', Hans A. Bechtel ${ }^{5}$, Amy A. Cordones ${ }^{1}$, Philipp Sperling ${ }^{1,6}$, Sven Toleikis ${ }^{7}$, Jan F. Kern (1D ${ }^{1}$, Stefan P. Moeller ${ }^{1}$, Siegfried H. Glenzer ${ }^{1}$ \& Daniel P. DePonte ${ }^{1}$

Correction to: Nature Communications; https://doi.org/10.1038/s41467-018-03696-w; published online 10 April 2018.

The original version of this Article omitted the following from the Acknowledgements:

'P.B. was funded by the ELI Extreme Light Infrastructure Phase 2 (CZ.02.1.01/0.0/0.0/15008/0000162) from the European Regional Development Fund and the EUCALL project funded from the EU Horizon 2020 research and innovation programme under grant agreement No 654220,' which replaces the previous 'P.B. was funded by the ELI Extreme Light Infrastructure Phase 2 (CZ.02.1.01/0.0/ 0.0/15008/0000162) from the European Regional Development Fund.'

This has been corrected in both the PDF and HTML versions of the Article.

Published online: 17 July 2018

Open Access This article is licensed under a Creative Commons Attribution 4.0 International License, which permits use, sharing, adaptation, distribution and reproduction in any medium or format, as long as you give appropriate credit to the original author(s) and the source, provide a link to the Creative Commons license, and indicate if changes were made. The images or other third party material in this article are included in the article's Creative Commons license, unless indicated otherwise in a credit line to the material. If material is not included in the article's Creative Commons license and your intended use is not permitted by statutory regulation or exceeds the permitted use, you will need to obtain permission directly from the copyright holder. To view a copy of this license, visit http://creativecommons.org/licenses/by/4.0/.
}

(C) The Author(s) 2018

\footnotetext{
${ }^{1}$ SLAC National Accelerator Laboratory, Menlo Park, CA 94720, USA. ${ }^{2}$ ELI Beamlines, Institute of Physics of the Czech Academy of Sciences, Na Slovance 2, Prague 18221, Czech Republic. ${ }^{3}$ Thayer School of Engineering, Dartmouth College, 14 Engineering Dr, Hanover, NH 03755, USA. ${ }^{4}$ Department of Electrical and Computer Engineering, University of Alberta, Edmonton, AB T6G 1H9, Canada. ${ }^{5}$ Advanced Light Source, Lawrence Berkeley National Laboratory, Berkeley, CA 94720, USA. ${ }^{6}$ European X-Ray Free-Electron Laser Facility GmbH, Schenefeld 22869, Germany. ${ }^{7}$ Deutsches Elektronen-Synchrotron, DESY, Notkestraße 85, Hamburg D-22607, Germany. Correspondence and requests for materials should be addressed to J.D.K.(email: koralek@slac.stanford.edu)
} 